\title{
How Can Software SMEs Become Medical Device Software SMEs
}

\author{
Fergal Mc Caffery, Valentine Casey \& Martin Mc Hugh \\ Regulated Software Research Group, \\ Dundalk Institute of Technology \& Lero, Dundalk, \\ Co. Louth, \\ Ireland
}

\{fergal.mccaffery, val.casey, Martin.McHugh\}@dkit.ie

Keywords: Medical device software, Software development, Software process improvement and assessment, Medi SPICE, Medical device standards, SME's.

\begin{abstract}
The amount of software content within medical devices has grown considerably over recent years and will continue to do so as the level of complexity of medical devices increase. This is driven by the fact that software is introduced to produce sophisticated medical devices that would not be possible using only hardware. This therefore presents opportunities for software development SMEs to become medical device software development organisations. However, some obstacles need to be addressed and overcome in order to make the transition from being a generic software development organisation to becoming a medical device software development organisation. This paper describes these obstacles and how research that is currently being performed within the Regulated Software Research Group in Dundalk Institute of Technology may be used to assist with this transition.
\end{abstract}

\section{Introduction - Background to Medical Device Software Development}

Today software is an increasingly important component of medical devices, as it enables often complex functional changes to be implemented without necessitating changes to the hardware [1]. As a consequence of the increasing demands for greater functionality within medical devices, the complexity of medical device software development also continues to increase [2]. This has resulted in increased demand for appropriate traceability and risk management processes and tools.

It is very important that highly effective software development practices are in place within medical device companies due to the safety-critical nature of medical device software. In order to sell their products medical device companies must comply with the regulatory requirements of the countries in which they wish to market their devices [3]. Governments have established regulatory bodies to tackle these issues 
whose role is to define regulatory systems for medical devices and to ensure that only safe medical devices are placed on the market [4].

While regulatory bodies offer some guidance on what software activities must be performed, no specific method for performing these activities is provided [5]. In this context in the United States (US), the Food and Drug Administration (FDA) Center for Devices and Radiological Health $(\mathrm{CDRH})$ has published guidance papers which include risk-based activities to be performed during software validation [6], premarket submission [7] and when using off-the-shelf software in a medical device [8]. Although the $\mathrm{CDRH}$ guidance documents provide information on which software activities should be performed, they do not enforce any specific method for performing these activities. This can result in medical device software companies failing to comply with the expected requirements.

To help address this situation a decision was taken by the medical device industry to recognize ISO/IEC 12207:1995 [9] (a general software engineering lifecycle process standard) as suitable for general medical device software development. Subsequently the Association for the Advancement of Medical Instrumentation (AAMI) software committee carefully reviewed ISO/IEC 12207:1995 and identified a number of shortcomings due to the fact that it was a generic standard. This resulted in the decision to create a new standard which was domain specific to medical device software development. The AAMI did not discard the work done with the ISO/IEC 12207:1995 and used it as the foundation for their new standard "AAMI SW68, Medical device software - Software lifecycle processes" [10]. In 2006, a new standard IEC 62304 [11] was released that was based on the AAMI SW68 standard.

The Council of the European Communities published in 1993 the Directive 93/42/EEC (1993) [12],"the Medical Device Directive" (MDD), on medical devices. The MDD is intended to ensure the safety of medical devices placed on the market in the European Union (EU), and has the backing of national legislation in member states. Amendments to this directive occurred via Directives 2000/70/EC (2000) [13], 2001/104/EC (2001) [14], 2003/32/EC (2003) [15], and 2007/47/EC (2007) [16].

Whenever we mention medical device guidelines within this paper we refer to the following medical device standards and guidelines: IEC 62304, FDA, the MDD, ISO 14971 [17], EN 60601-4 [18], TIR 32 [19], IEC TR 80002-1[20], IEC 62366 [21],GAMP 5 [22], IEC/TR 61508 [23], ISO 13485[24] and IEC 60812 [25].

The remainder of this paper is structured as follows: Section 2 considers the reasons why software Small and Medium sized Enterprises (SMEs) are interested in becoming medical device SMEs and the challenges they face. In section 3 recent changes made by the EU and FDA regarding medical device software are discussed. Section 4 outlines the research currently being undertaken by the Regulated Software Research Group (RSRG) in Dundalk Institute of Technology which is of particular value to assist SMEs to develop medical device software. 
Section 5 discusses what initial steps can be taken by SMEs when embarking on medical device software development. A conclusion is provided in section 6 and future work is outlined.

\section{Why Software SMEs are Interested in Becoming Medical Device SMEs}

Software development SMEs are currently becoming medical device software development SMEs for two main reasons. The first of these is medical devices are becoming increasingly more complex and software facilitates increased functionality without the necessity to replace hardware. Therefore, due to growth in this area for this type of software there is an opportunity for software development SMEs to become medical device software development SMEs. The second reason is that software development SMEs currently developing health related software applications may now be actually developing medical device software. This is due to the most recent revision of the of Medical Device Directive [16], which states that standalone software may now be defined as a medical device.

The challenge both types of organizations face is that to become a medical device software SME requires that software be developed in a regulatory compliant manner. This is essential as in order for a medical device to be marketed it is first necessary to achieve regulatory approval for the device in the region where it will be sold. This therefore means that such organizations need to become aware of what developing regulatory compliant software means and how they will have to change their current software development processes in order to fulfill this requirement. The starting point for such organizations is to become aware of the relevant regulations.

\section{Changes Impacting Medical Device Software Development}

As medical devices are safety critical, they are subject to stringent regulations before they can be approved for use. Within the US medical devices must be approved by the FDA and likewise, medical devices for use within the EU must carry a CE conformance mark. This is awarded by notified bodies in each country. The most recent changes in the regulations, with regard to medical device software development, is the European MDD (2007/47/EC) [16] and the FDA Final Rule on Medical Device Data Systems [26].

On March 21st 2010, the MDD (2007/47/EC) came into force in the EU. This directive amends the MDD (93/42/EEC) [12], the Active Implantable Medical Device directive (90/385/EEC) [27] and the Biocides directive (98/8/EC) [28]. The most 
significant amendment within the MDD (2007/47/EC) is the provision for standalone software to be used as an active medical device. The MDD (2007/47/EC) Annex IX Section 1.4 states: "stand-alone software is considered to be an active medical device". It defines an active medical device as "any medical device operation which depends on a source of electrical energy or any source of power other than that generated by the human body or gravity"[16].

Consequently, standalone medical device software is now subject to regulation, independent of the hardware on which it resides. As software can now be seen as the only component of a medical device, improved guidance is needed in the development of this type of software to achieve regulatory compliance. While the MDD provides medical device manufacturers with a list of harmonized standards which can be used during development to aid in achieving regulatory compliance. However, no harmonized standards exist that can provide full guidance in the development of standalone software as an active medical device. The FDA does not provide software or standalone software specific regulations. They regulate medical devices and the elements that are included as part of that device, with software being regulated in this context. To help address this the FDA provide relevant guidance documents to assist with medical device software development [7], [6], [8].

On April 18th 2011, the FDA Final Rule reclassifying Medical Device Data systems (MDDS) as Class I medical devices became effective [26]. MDDS's are off-the-shelf or custom software or hardware products used alone, or in combination, that display unaltered medical device data or transfer, store or convert medical device data for future use, in accordance with a pre-set specification. Prior to the release of this final rule, MDDS were classified as Class III devices or as an accessory to the parent device, requiring the greatest amount of scrutiny before approval could be awarded. Whilst this ruling will make it simpler for companies developing MDDS software, it will also ring fence those which were previously beyond the net of regulatory requirements and make them subject to regulation. In addition, as part of this final rule a caveat was added to exclude devices that are used to actively monitor patients from being included as a MDDS. These devices remain classified as accessories and must undergo the same amount of regulatory conformance as the parent device to which they are connected [29]. Also standalone software such as Electronic Health Records (EHR) and Computerized Physician Order Entry (CPOE) fall outside of the scope of being defined as MDDS.

\section{Research to Help SMEs Develop Medical Device Software}

The RSRG was established in Dundalk Institute of Technology in February 2008. The research undertaken by the RSRG is focused on the development of an 
international Software Process Improvement (SPI) framework for the medical device industry. The objective of this framework is to provide a key enabler of best practice for the sector.

The RSRG have undertaken a multi-faceted approach to establishing this framework including the examination of best practice from other safety-critical domains and determining how best practice SPI models can be successfully mapped onto regulatory frameworks. This has taken place through close cooperation with the medical device industry, relevant international standards bodies and the software process improvement community. A key element of this research is the use of empirical studies of industrial practice which has been utilized to inform theory. To undertake this work the RSRG have adopted a range of quantitative and qualitative research methodologies including experiments, quantitative analysis of data sets, case studies, action research and grounded theory to provide a rich analysis of the domain.

The main focus of the RSRG is to support the growth of the medical device software industry within Ireland. However, this does not mean that their research has been restricted to Ireland, in fact the RSRG collaborates closely with international medical device organizations and SPI researchers. In particular, the RSRG is working as part of an international working group to revise the International Standard for Software Medical Device Software Lifecycle Processes (IEC 62304) and also closely with the Spice User Group to develop Medi SPICE [30]. Whilst the main deliverable for the RSRG is Medi SPICE they have also developed a number of lightweight assessment methods to assist software SMEs to become medical device software SMEs. The following subsections describe Medi SPICE and the lightweight assessment methods Med-Trace, Med-Adept and Medi SPICE-Adept.

\subsection{Medi SPICE}

Medi SPICE [30], [5] is a process assessment and improvement model which is domain specific to medical device software development and incorporates regulatory compliance. The results of a Medi SPICE assessment may be used to indicate the state of a medical device suppliers software practices in relation to the regulatory requirements of the industry, and identify areas for process improvement. The results of an assessment may also be used as a criterion for supplier selection.

Medi SPICE is based on ISO/IEC 15504-5 [31] and provides coverage of the medical device software regulations. Like ISO/IEC 15504-5 and Automotive SPICE [32] it contains both a Process Reference Model (PRM) and Process Assessment Model (PAM) containing processes that provide comprehensive coverage of the FDA and 
European Council directives, and associated standards (e.g. ISO 14971, ISO 13485, IEC TR 80002-1, IEC 62304) for the complete software development lifecycle.

The overall objective of Medi SPICE is to provide a conformity assessment scheme to support first, second or third party assessment results that may be recognized by the regulatory bodies. The PRM and PAM of the Medi SPICE assessment model is derived from relevant ISO/IEC 15504-5 processes as they are all applicable to the development of safety-critical medical device software. As the IEC 62304 standard contains the medical device software lifecycle processes that have to be adhered to in order to achieve medical device regulatory compliance, a key objective is to provide coverage of all processes that are either included in or referenced from IEC 62304 and its associated standards.

The Medi SPICE PRM and PAM is being released in phases and consists of a defined set of software processes that contain a comprehensive set of specific practices which when utilized assist medical device software development organizations to fulfill the regulatory guidelines and standards of the medical device industry. It also addresses the requirements for process assessment and can be utilized to facilitate process improvement.

\subsection{Med-Trace}

As traceability is central to the development of regulatory compliant software the RSRG decided to develop an assessment method specifically to assist companies to adhere to the traceability aspects of the medical device software standards. Emanating from the Adept method [33], previously developed by the authors, and based on CMMI [34] and ISO/IEC 15504-5 software process reference models, Med-Trace is a lightweight assessment method that provides a means of assessing the capability of an organization in relation to medical device software traceability. Med-Trace enables software development organizations to gain an appreciation of the fundamental traceability best practices based on the software engineering traceability literature, software engineering process models (CMMI $\Theta$, ISO/IEC 15504-5), and the medical device software guidelines and standards. Med-Trace may be used to diagnose an organization's weaknesses and strengths with relation to their medical device software development traceability practices.

Med-Trace is composed of 8 stages. The assessment team typically consists of two assessors who conduct the assessment between them. Stage 1, involves a preliminary meeting between the assessment team and the company wishing to undertake a Med-Trace assessment. At stage 2, the lead assessor presents an overview of the Med-Trace assessment to members of the organization who will be involved in subsequent stages. Stage 3, provides a brief insight into project documentation. The first 3 stages are normally performed on the company's premises, but the sample documentation collected in stage 3 is sometimes taken off- 
site as it can then be used to assist with the generation of additional questions for stage 4.

During stage 4, the assessment team return onsite and key staff members from the organization are interviewed. A set of scripted questions are utilized as a basis for these interviews. These questions are based on the software traceability literature, traceability practices within the CMMI® and ISO/IEC 15504-5 models, and traceability practices that are required by the medical device industry. Additional questions may be asked based on the review of the documentation outlined in stage 3.

Stage 5, is a collaborative exercise between the assessors to develop the findings report using interview notes. Stage 6, involves presenting the findings report to participating staff in the organization. Stage 7 , entails collaborating with staff to develop a pathway towards achieving highly effective and regulatory compliant traceability practices. The findings report provides guidance to the assessed company and focuses on practices that provide the greatest benefit in terms of the company's business goals, in addition to their quality and compliance needs. At Stage 8, the assessed company is revisited approximately 3 months after the completion of stage 7 and their progress is reviewed against the recommended improvement path. The outcome of this stage is an updated improvement path and a final report detailing the progress that has been accomplished along with additional recommendations.

\subsection{Med-Adept}

The Adept method [33] was previously developed to provide a lightweight assessment of software processes from CMMI® and ISO/IEC 15504-5 and was not domain specific. The Adept method was then integrated with practices specified in medical device regulatory guidelines and standards to produce Med-Adept. MedAdept [35] is an assessment method that provides a means of assessing the software engineering capability for processes in relation to medical device software (both application and embedded software).

Med-Adept enables software development organisations to gain an appreciation of the fundamental processes from CMMI®, ISO/IEC 15504-5 and IEC 62304 (including additional practices required by other medical device guidelines and standards) through diagnosing strengths and weaknesses in their software development practices. Med-Adept was designed to adhere to 8 of the 10 criteria outlined by Anacleto et al. [36], for the development of lightweight assessment methods: low cost, detailed description of the assessment process, guidance for process selection, detailed definition of the assessment model, support for identification of risks and improvement suggestions, conformity with ISO/IEC 155045 , no specific software engineering knowledge required from companies' representatives, and tool support is provided. The two exceptions to the criteria 
outlined by Anacleto et al. [36] are that no support is provided for high-level process modeling and only the authors currently have access to the method. Med-Adept also inherits the following requirements from Adept: improvement is more important than certification, a rating is not required, preparation time required by the company is minimised; assessment time is minimized, and companies should be enabled to select assessment in the process areas that are most relevant to their business goals.

The main aims of Med-Adept are to either encourage non-medical device software development organisations to develop software for the medical device industry or to improve the software development processes within existing medical device software development organizations. However, the Med-Adept method also provides an ideal opportunity to educate software development organisations in terms of generic SPI. Therefore, the assessment would still have value even if the assessed software development company did not intend becoming a medical device software development company in the future. Consequently, Med-Adept provides medical device specific and non-medical device specific recommendations. Assessed companies are also supplied with feedback in relation to both CMMI® and ISO/IEC 15504-5 which enables such companies to decide whether they wish to follow a CMMI® or an ISO/IEC 15504-5 improvement path. Med-Adept provides the assessed company with a findings document presented in terms of processes from CMMI®, ISO/IEC 15504-5 and practices required by medical device software standards and regulations (with a particular focus on IEC 62304).

Med-Adept is composed of 8 stages which are similar to those in Adept and MedTrace. The assessment team consists of two assessors who conduct the assessment between them. Stage 1 involves a preliminary meeting between the assessment team and the software company wishing to undergo a software process assessment. The assessment team discuss the main drivers for the company embarking on a Med-Adept assessment and establish whether the company is interested in developing software for the medical device industry. During stage 2 the lead assessor provides an overview of Med-Adept for members of the assessed organisation who will be involved in subsequent stages. This session is used to remove any concerns that individuals may have.

Stage 3 provides a brief review of project documentation. However, the primary source of data for Med-Adept is through a series of process interviews conducted during stage 4 . In this stage key staff members from the assessed organisation are interviewed. There is an interview for each process. Each interview is scheduled to last approximately 1.5 hours. To enable stage 4 to be completed within 1 day the scope of a single Med-Adept assessment is restricted to 4 processes. Each interview involves two assessors and at least one representative from the company. Stage 5 is a collaborative exercise between the assessors to develop the findings report using interview notes for each of the assessed processes. The resultant findings report 
consists of a list of strengths, issues and suggested actions for each of the assessed processes.

Stage 6, involves presenting the findings report to participating staff in the organisation. Stage 7, involves collaborating with staff to develop a roadmap. This provides guidance to the assessed company presenting practices that can provide the greatest benefit in terms of the company's business goals. Companies wishing to develop software for the medical device industry are recommended to focus on establishing working practices that will assist them to fulfil the medical device regulations. Stage 8, involves revisiting the assessed company approximately 3 to 6 months after the completion of stage 7 and reviewing progress against the SPI path. The outcome of this stage is an updated SPI path and a final report detailing the progress that has been accomplished along with additional recommendations. This stage provides feedback and assistance to the assessed company after a period of time and also assists in compiling research material in terms of SPI experiences.

As Med-Adept is based on the Adept method, existing Adept questions were used as the foundation for the Med-Adept method. Questions were added to enable coverage of medical device regulations. Even though each assessment component adopts a $\mathrm{CMMI} \circledast \mathrm{B}$ process area name, it also contains questions providing coverage of relevant ISO/IEC 15504-5 processes and medical device standards and regulations.

Of the 12 Adept processes, 11 had medical device regulatory questions added for Med-Adept the exception being Measurement and Analysis which cannot be mapped against the processes of IEC 62304. Additionally, the existing Adept processes (which Med-Adept is founded on) did not provide coverage of 5 IEC 62304 processes (Software Release, Software Maintenance, Software Problem Solution, Documentation, Software Safety Classification). Therefore, the current version of Med-Adept also does not provide coverage of these processes. Additionally, it should also be noted that the current release of Med-Adept does not include a Risk Management process.

Therefore Med-Adept does not provide complete coverage of all the medical device regulations. However, the main aim of Med-Adept is not to provide comprehensive coverage of medical device regulations, but rather to assist organisations to improve their software practices and to encourage organizations to develop medical device software. To encourage the uptake of the Med-Adept assessment by software SMEs, on-site interviewing is restricted to one day thus minimizing the time and cost associated with the assessment.

\subsection{Medi SPICE-Adept}


The RSRG are currently developing a new assessment method (Medi SPICE-Adept) that will enable lightweight assessments to be performed against the Medi SPICE PAM. Medi SPICE-Adept follows the same structure as Med-Adept but with several key differences:

- $\quad$ Different processes then Med-Adept - Due to changes in the configuration of the processes that makeup Medi SPICE, Med Adept does not provide coverage of all Medi SPICE processes. Medi SPICE-Adept will provide coverage of all Medi SPICE processes but currently it consists of only 10 processes: Configuration Management, Change Request Management, Software Requirements Elicitation, Systems Requirements Analysis, Software Requirements Analysis, Software Construction, Software Integration, Software Testing, Verification, Validation

- $\quad$ Not based on $\mathrm{CMMI} B$ - Whereas, Med-Adept was founded on CMMI® questions, ISO/IEC 15504-5 and regulatory processes Medi SPICE-Adept is not based on $C M M I \circledast$ and does not include $C M M I \circledast$ questions. Medi SPICE-Adept is instead based on Medi SPICE processes.

- Includes agile and lean questions - Medi SPICE-Adept, unlike Med-Adept contains questions that refer to agile and lean practices. The output from an assessment is a set of strengths, issues and recommendations. As companies wish to increase the efficiency of their software development practices it was therefore considered essential to include agile and lean practices as part of the assessment.

- Reflects changes in latest directives' and standards - Contains processes that are in-line with the latest changes in the MDD and the FDA rules. Med-Adept was initially developed to provide assessment of Medi SPICE processes that were aligned with ISO 12207:1995, however, both IEC 62304 and ISO/IEC 15504-5 are now being revised to align with ISO 12207:2008. It is therefore important that Medi SPICE is also revised to align with ISO 12207:2008. Therefore, Medi SPICE-Adept provides coverage of the revised Medi SPICE processes.

- $\quad$ May be performed over a number of days. Whereas a Med-Adept assessment typically involved only one day of on-site interviews, a Medi SPICE-Adept assessment currently consists of 2 days of on-site interviews and provides coverage of 10 process areas. However, in the future we plan to extend the scope of the assessment to include all 38 Medi SPICE processes and 10 subprocesses. Although, it will still be possible for the organization to be assessed in as few 
processes as they wish and in such cases the on-site interviews may be completed in one day.

\section{What initial steps may be taken by SMEs}

The first step for a company is to discover if their software comes under the umbrella of a medical device as defined by either the revision of the European Council's MDD or the FDA's MDDS. The second step will then be to become aware of what standards need to be adhered to. This can be quite a time-consuming task for organizations as there are many different standards and it is difficult to determine the complete list of appropriate standards and the relationship between the different standards.

The third step is for an organization to benchmark the state of their current software development processes against recommended best practices for medical device software development. In relation to this the author's recommend adopting a MedTrace assessment to gain an understanding of how the traceability aspects of their software development processes could be improved to assist them to achieve regulatory compliance. Additionally, the author's also recommend implementing a Medi SPICE-Adept assessment to gain an understanding as to the set of strengths and issues of an organization's current software development processes in relation to developing regulatory compliant medical device software. The output from implementing both Med-Trace and Medi SPICE-Adept will be a set of recommendations that will provide a roadmap to put an organization on the correct path towards developing medical device software using compliant processes. It should be noted that if an organization decides to implement a Medi SPICE-Adept assessment this would remove the need to perform step 2 as this assessment is based on Medi SPICE which provides seamless alignment across all the required medical device software standards. It will then be important for the organization to prioritize the recommendations and start to implement them within their organization - this should be performed by implementing a few recommendations at a time as opposed to everything at once.

After implementing the recommendations over a period of time the organization should then embark on another Medi SPICE-Adept and/or Med-Trace assessment to determine the level of improvement in relation to their software development processes. The output from this follow-up assessment will either be another set of recommendations or an indication that adequate processes are now in place for the organization to contact the national notified body for an official certification.

\section{Conclusion}

The growing requirement for medical device software and the opportunities this provides for software SMEs continues to increase. The necessity for regulatory compliance and the lack of clear guidance are often cited as barriers to entry to this expanding market. Organizations that are currently developing medical device 
software are mainly focused on achieving regulatory compliance rather than also improving their development process and increasing their efficiency. For both of these situations the research undertaken by the RSRG is of particular value. Medi SPICE is a process assessment and improvement model which is domain specific to medical device software development. It has been developed to address the specific requirements of medical device software companies. Med-Trace and Medi SPICEAdept (based on Medi SPICE and Med-Adept) are lightweight assessment methods which provide effective mechanisms to assist software SMEs to enable them to put processes in place that will enable them to perform medical device software development. The RSRG plan to continue to build on its extensive experience and knowledge in this area. It is envisaged this will include the development of additional assessment methods and tools in the future as opportunities for innovation and improvement arise.

\section{Acknowledgements.}

This research is supported by the Science Foundation Ireland (SFI) Stokes Lectureship Programme, grant number 07/SK/I1299, the SFI Principal Investigator Programme, grant number 08/IN.1/I2030 (the funding of this project was awarded by Science Foundation Ireland under a co-funding initiative by the Irish Government and European Regional Development Fund), and supported in part by Lero - the Irish Software Engineering Research Centre (http://www.lero.ie) grant 03/CE2/I303_1.

\section{References}

1. Lee, I., G. Pappas, R. Cleaveland, J. Hatcliff, B. Krogh, P. Lee, H. Rubin, and L. Sha, High-Confidence Medical Device Software And Systems. Computer, 2006. 39(4): p. 33 - 38.

2. Rakitin, R., Coping with defective software in medical devices. Computer, 2006. 39 (4): p. $40-45$.

3. Burton, J., F. Mc Caffery, and I. Richardson. A risk management capability model for use in medical device companies. in International Workshop on Software quality (WoSQ '06). 2006. Shanghai, China: ACM.

4. Mc Caffrey, F., J. Burton, V. Casey, and A. Doring, Software Process Improvement in the Medical Device Industry, in Encyclopedia of Software Engineering, P. Laplante, Editor. 2010, CRC Press Francis Taylor Group: New York. p. 528 - 540.

5. McCaffery, F., A. Dorling, and V. Casey. Medi SPICE: An Update. in International Conference on Software Process Improvement and Capability Determinations (SPICE). 2010. Pisa, Italy: Edizioni ETS. 
6. US FDA Center for Devices and Radiological Health, General Principles of Software Validation; Final Guidance for Industry and FDA Staff. 2002, CDRH: Rockville.

7. US FDA Center for Devices and Radiological Health, Guidance for the Content of Premarket Submissions for Software Contained in Medical Devices. 2005, CDRH: Rockville.

8. US FDA Center for Devices and Radiological Health, Off-The-Shelf Software Use in Medical Devices; Guidance for Industry, medical device Reviewers and Compliance. 1999, CDRH: Rockville.

9. ISO/IEC 12207:1995, Information Technology — Software life Cycle Processes. 1995, ISO: Geneva, Switzerland.

10. ANSI/AAMI SW68:2001, Medical device software - Software life cycle processes. 2001, AMMl: Arlington.

11. IEC 62304:2006, Medical device software-Software life cycle processes. 2006, IEC: Geneva, Switzerland.

12. European Council, Council Directive 93/42/EEC Concerning Medical Devices. 1993, Official Journal of The European Communities: Luxembourg.

13. European Council, Council Directive 2000/70/EC (Amendment). 2000, Official Journal of The European Union: Luxembourg.

14. European Council, Council Directive 2001/104/EC (Amendment). 2001, Official Journal of The European Union: Luxembourg.

15. European Council, Council Directive 2003/32/EC (Amendment). 2003, Official Journal of The European Union: Luxembourg.

16. European Council, Council Directive 2007/47/EC (Amendment). 2007, Official Journal of The European Union: Luxembourg.

17. ISO 14971:2007, Medical Devices - Application of risk management to medical devices. 2007, ISO: Geneva.

18. BS EN 60601-1-4:2000, Medical Electrical Equipment, Part 1 - General requirements for safety. $2000, \mathrm{BSI}$ : London.

19. AAMI TIR32:2004, Medical device software risk management. 2005, AAMI: Arlington.

20. IEC/TR 80002-1:2009, Medical device software Part 1: Guidance on the application of ISO 14971 to medical device software. 2009, BSI: London. 
21. IEC 62366:2007, Medical devices - Application of usability engineering to medical devices. 2007, IEC: Geneva, Switzerland.

22. GAMP 5:2008, A Risk-Based Approach to Compliant GxP Computerized System. 2008, ISPE: Florida.

23. IEC/TR 61508:2005, Functional safety of electrical/electronic/ programmable electronic safety related systems. 2005, BSI: London.

24. ISO 13485:2003, Medical devices - Quality management systems Requirements for regulatory purposes. 2003, ISO: Geneva, Switzerland.

25. IEC 60812:2006, Analysis technique for system reliability - Procedure for failure modes and effects analysis (FMEA). 2006, IEC: Geneva, Switzerland.

26. US FDA, 21 CFR Part 880 Medical Devices; Medical Device Data Systems Final Rule. Federal Register, 2011. 76 (31): p. 8637 - 8649.

27. European Council, Council Directive 90/385/EEC On the approximation of the laws of the Members States relating to active implantable medical devices. 1990, Official Journal of The European Communities]: Luxembourg.

28. European Council, Council Directive 98/8/EC Concerning the placing of biocidal products on the market. 1998, Luxembourg: Official Journal of The European Communities.

29. Thompson, B., FDA Regulations of Mobile Health, in Mobi Health News. 2010, Chester Street Publishing. p. 51.

30. McCaffery, F. and A. Dorling. Medi SPICE: An Overview. in International Conference on Software Process Improvement and Capability Determinations (SPICE). 2009. Turku, Finland.

31. ISO/IEC 15504-5:2006, Information technology — Process Assessment - Part 5: An Exemplar Process Assessment Model. 2006, ISO: Geneva, Switzerland.

32. Automotive SPICE Process Assessment. 21 August 2005: SIG.

33. Mc Caffery, F., I. Richardson, and G. Coleman. Adept - A Software Process Appraisal Method for Small to Medium-sized Irish Software Development Organisations. in European Systems \& Software Process Improvement and Innovation (EuroSPI 2006). 2006. Joensuu, Finland.

34. CMMI Product Team, Capability Maturity Model® Integration for Development Version 1.2. 2006, Software Engineering Institute.

35. Mc Caffery, F. and V. Casey. Med-Adept: A Lightweight Assessment Method for the Irish Medical Device Software Industry. in European Systems \& Software 
Process Improvement and Innovation Conference, (EuroSPI). 2010. Grenoble, France.

36. Anacleto, A., C.G. von Wangenheim, C.F. Salviano, and R. Savi. Experiences gained from applying ISO/IEC 15504 to small software companies in Brazil. in 4th International SPICE Conference on Process Assessment and Improvement. 2004. Lisbon, Portugal. 